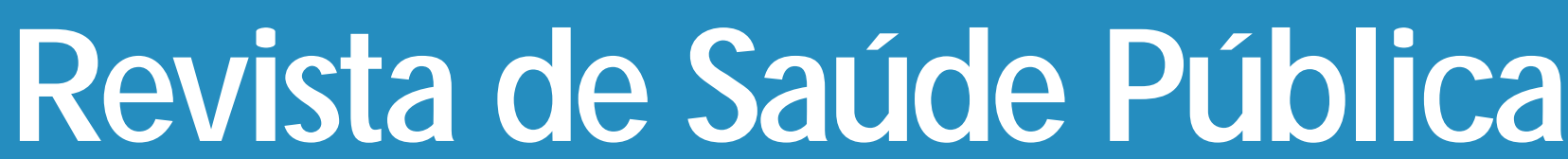

$\begin{array}{llllllll} & \mathbf{O} & \mathbf{U} & \mathbf{R} & \mathbf{N} & \mathbf{A} & \mathbf{L}\end{array}$

O F

$\mathbf{P} \mathbf{U}$

B L I C

H E A L T H

\title{
Comparação de dados epidemiológicos da tuberculose pulmonar em Sorocaba, SP, Brasil, em uma década (1986 - 1996)
}

Some epidemiological data on pulmonary tuberculosis in an urban area in Brazil, in comparison with in one decade (1986 - 1996)

José R. P. P. Job, Paulo E. B. S. Prado*, Sérgio Vranjac* e Paulo C. D uarte*

Departamento de Medicina da Faculdade de M edicina de Sorocaba da Pontifícia Universidade Católica de São Paulo (D M FM S/PU C-SP). Sorocaba, SP - Brasil (U.R.P.P.J.)

JO B José R. P. P. , Paulo E. B. S. Prado*, Sérgio Vranjac* e Paulo C. Duarte* Comparação de dados epidemiológicos da tuberculose pulmonar em Sorocaba, SP, Brasil, em uma década (1986 - 1996) Rev. Saúde Pública, 32 (6): $596-7,1998$

(c) Copyright Faculdade de Saúde Pública da USP. Proibida a reprodução mesmo que parcial sem a devida autorização do Editor Científico. Proibida a utilização de matérias para fins comerciais. All rights reserved. 


\title{
Comparação de dados epidemiológicos da tuberculose pulmonar em Sorocaba, SP, Brasil, em uma década (1986 - 1996)
}

\section{Some epidemiological data on pulmonary tuberculosis in an urban area in Brazil, in comparison with in one decade (1986 - 1996)}

\author{
José R. P. P. Job, Paulo E. B. S. Prado*, Sérgio Vranjac* e Paulo C. D uarte* \\ Departamento de Medicina da Faculdade de Medicina de Sorocaba da Pontifícia Universidade \\ Católica de São Paulo (DM FM S/PU C-SP). Sorocaba, SP - Brasil (J.R.P.P.J.)
}

\begin{abstract}
Resumo
Em 50 doentes matriculados para o tratamento de tuberculose pulmonar, foram estudadas as informações que antecederam o diagnóstico da doença tais como: sintomatologia antes do diagnóstico, tempo decorrido entre o aparecimento dos sintomas e a procura de assistência médica, tempo decorrido entre o início dos sintomas e o início do tratamento e presença ou não de co-infecção com HIV. Nos últimos dez anos não houve alteração significativa em relação ao tempo de identificação dos casos novos de tuberculose e o início do tratamento.
\end{abstract}

Tuberculose pulmonar, epidemiologia. Incidência. Síndrome de imuno-deficiência adquirida. Centros de Saúde.

\begin{abstract}
Pulmonary tuberculosis is a serious public health problem in Brazil. Data were collected relating to the patients' health before diagnosis and also to the time lag between this diagnosis and the beginning of specific treatment over a period of three months. Fifty patients suffering from pulmonary tuberculosis were questioned about: symptomatology before diagnosis, lapse between the occurrence of the first symptoms and the beginning of treatment and relationship with AIDS. There had been no significant change in the time-lag between the time of discovery of new cases and the beginning of treatment, over the last ten years.
\end{abstract}

Tuberculosis, pulmonary, epidemiology. Incidency. Acquired immuno-deficiency syndrome. Health Centers.

*Acadêmicos do DMFMS/PUC-SP.

Correspondência para/Correspondence to: José R.P.P.Job - Praça Dr José Ermírio de Moraes,290 - Jardim Faculdade - 18030-000 - Sorocaba, SP Brasil. Fax: (015) 232-0445.

Edição subvencionada pela FAPESP (Processo no 97/09815-2).

Recebido em 24.3.1998. Aprovado em 13.7.1998. 
A tuberculose pulmonar ainda hoje, é um problema de saúde pública muito grave ${ }^{3}$. Há uma década era utilizada como indicador de subdesenvolvimento, mas hoje com a disseminação da SIDA pelo mundo, tornou-se a terceira doença oportunista mais freqüente em pacientes HIV positivos.

No mundo, a Organização Mundial de Saúde acredita que no ano 2000 haverá cerca de 163 casos por 100 mil habitantes, que acarretarão cerca de 3,5 milhões de mortes ${ }^{1}$. No Estado de São Paulo a incidência de tuberculose é da ordem de 57,6 casos por 100 mil habitantes ${ }^{2}$.

Sabe-se que o esquema terapêutico é capaz de curar cerca de $95 \%$ dos casos ${ }^{2}$. Por isso a demora no diagnóstico faz com que a cadeia de transmissão prolongue-se por um tempo desnecessário, acarretando o contágio e surgimento.

Pretende-se contribuir para a caracterização do perfil sintomático do doente com tuberculose que procura o Centro de Saúde, e comparar os dados obtidos com os conseguidos há 10 anos em trabalho semelhante ${ }^{3}$.

No período compreendido entre 6/5/96 e 8/8/96 foram entrevistados 50 pacientes seqüenciais ou os seus responsáveis, de ambos os sexos, com tuberculose pulmonar que espontaneamente procuraram o Centro de Saúde I do Departamento Regional de Saúde de Sorocaba. Foi aplicado questionário com a finalidade de se obter informações sobre a sintomatologia antes do diagnóstico, tempo decorrido entre o início dos sintomas e a procura de serviço médico e o início da terapêutica, além da presença ou não de co-infecção com HIV.

Os dados obtidos foram comparados com os de uma pesquisa semelhante, realizada há 10 anos $^{3}$, através dos testes estatísticos do qui-quadrado.

Os resultados encontrados mostraram que a tosse foi o sintoma mais frequiente $(96 \%)$ seguido pela expectoração ( $84 \%$ ), sudorese (84\%) e emagrecimento $(84 \%)$, febre $(82 \%)$, dor torácica $(76 \%)$, dispnéia $(68 \%)$ e hemoptise (28\%).Em $20 \%$ dos pacientes o tempo decorrido entre o início dos sintomas e a procura da assistência médica foi maior do que 3 meses, em $8 \%$ entre 2 e 3 meses, em $28 \%$ entre 1 e 2 meses e em $44 \%$ menor do que 1 mês. Quando a terapêutica foi introduzida, $32 \%$ dos pacientes apresentavam sintomas há mais de 3 meses, $16 \%$ entre 2 e 3 meses, $22 \%$ entre 1 e 2 meses e $30 \%$ há menos de 1 mês. A co-infecção com HIV foi verificada em $18 \%$ dos pacientes.

A frequiência elevada de tosse demonstra que esse sintoma é o principal fator para se pensar no diagnóstico de tuberculose pulmonar, principalmente quando se trata de uma tosse com duração superior há 1 mês. Nesses casos a Campanha Nacional contra a Tuberculose ${ }^{1}$ indica a necessidade de realização de exame baciloscópico de escarro.
A co-infecção com HIV, a deterioração das condições socioeconômicas da população, o desemprego, o etilismo, o tabagismo e a falta de informação sobre a sintomatologia da doença, que leva à demora na procura do serviço médico, permitiram que houvesse um aumento significativo da frequiência dos sintomas; dor torácica e emagrecimento, quando relacionados a freqüência desses sintomas apresentados há 10 anos atrás ${ }^{3}$. (Tabela).

Tabela - Presença de sintomas nos 50 pacientes entrevistados, Centro de Saúde de Sorocaba, 1996.

\begin{tabular}{lllll}
\hline Sintomas & \multicolumn{2}{c}{1986} & \multicolumn{2}{c}{1996} \\
\hline Expectoração & $\mathrm{N}$ & $\%$ & $\mathrm{~N}$ & $\%$ \\
Febre & 34 & 68 & 42 & 84 \\
Hemoptise & 43 & 86 & 41 & 82 \\
Emagrecimento & 16 & 32 & 14 & 28 \\
Dispnéia & $\mathbf{3 3}$ & $\mathbf{6 6}$ & $\mathbf{4 2 *}$ & $\mathbf{8 4}$ \\
Tosse & 28 & 56 & 34 & 68 \\
Sudorese & 44 & 88 & 48 & 96 \\
Dor torácica & 37 & 74 & 42 & 84 \\
* Job e col. $^{3}$ (1986). Estatisticamente maior & $\mathbf{2 5}$ & $\mathbf{5 0}$ & $\mathbf{3 8 *}$ & $\mathbf{7 6}$ \\
\hline
\end{tabular}

* Job e col. ${ }^{3}$ (1986). Estatisticamente maior para $\mathrm{P}<0,05$

O fato de $32 \%$ dos pacientes apresentarem uma demora de cerca de 3 meses para a realização do diagnóstico levanta a suspeita sobre o provável despreparo, por parte dos médicos, em realizar o diagnóstico, assim como dos técnicos responsáveis pela realização do exame baciloscópico em fazer a correta interpretação do exame.

Do total de pacientes, $18 \%$ eram portadores de HIV, sendo portanto, de suma importância que se dê prioridade nos serviços de tuberculose, à identificação precoce dos pacientes HIV positivos ${ }^{4}$.

\section{REFERÊNCIAS}

1. CAMPOS, H. S. Tuberculose: um perigo real e crescente. $J B M, 70: 73-105,1996$.

2. JOB, J. R. P. P.; GOZZANO, J. O.A.; BERNARDES JUNIOR, O.R.; MIRALHES, O. J. C.; MIRANDA, M. A. P. Informações que antecederam o diagnóstico de tuberculose pulmonar e tempo decorrido até o início de tratamento em pacientes matriculados em centro de saúde, São Paulo (Brasil). Rev. Saúde Pública, 20:21-5, 1986.

3. KRITSKI, A.; DALCOLMO, M.; BIANCO, R.; MELO, F. F; PINTO, W. P.; SCHECHTHER, M.; CASTELO, A. Associação tuberculose e infecção pelo HIV no Brasil. Bol. Ofic. Sanit. Panam., 118:542-54, 1995.

4. MINISTÉRIO DA SAUUDE. Secretaria Nacional de Programas Especiais de Saúde. Divisão de Pneumologia Sanitária. Campanha Nacional Contra a Tuberculose. Controle da tuberculose: uma proposta de integração ensino-serviço. $2^{\mathrm{a}} \mathrm{ed}$. Rio de Janeiro, CNCT/NUTES, 1989. 